\title{
Ab initio lattice dynamics of nonconducting crystals by systematic fragmentation
}

\author{
Michael A. Collins ${ }^{a)}$ \\ Research School of Chemistry, Australian National University, Canberra, ACT 0200, Australia
}

(Received 16 February 2011; accepted 4 April 2011; published online 29 April 2011)

\begin{abstract}
A systematic method for approximating the $a b$ initio electronic energy of crystal lattices has been improved by the incorporation of long range electrostatic and dispersion interactions. The effect of these long range interactions on the optimization of the crystal structure is reported. The harmonic lattice dynamics have been evaluated to give phonon frequencies and neutron scattering intensities. Exemplary results are reported for diamond, silicon, and $\alpha$-quartz using Hartree-Fock, Möller-Plesset perturbation, and coupled-cluster levels of ab initio theory. () 2011 American Institute of Physics. [doi:10.1063/1.3581845]
\end{abstract}

\section{INTRODUCTION}

A number of groups ${ }^{1-18}$ have reported successful methods for estimating the $a b$ initio electronic energy (and other properties) of large molecules by breaking the molecule into fragments. In the simplest approaches, the energy of the whole molecule can be estimated from sums and differences of the energies of the fragments. The number of atoms in the fragments is unrelated to the size of the original molecule, but the number of fragments is proportional to the number of atoms in the whole molecule. Hence, the $a b$ initio computation time is only linearly proportional to the number of atoms in the molecule. A systematic method ${ }^{12,13}$ of this type has been presented which provides a hierarchy of fragmentations: producing fragments of larger size at higher "levels of fragmentation," which provide increasingly reliable estimates of the molecular energy (albeit at increasing cost).

This systematic fragmentation approach has been applied to the crystal structures of nonconducting materials, ${ }^{19}$ as well as to molecules. The infinite crystal structure is represented by a sum (and difference) of a relatively small number of molecular structures, which are infinitely periodically reproduced to give the whole crystal. The energy (and other properties) of the crystal (per unit cell) can be estimated from ab initio calculations on a few relatively small molecules. Hence, the computational cost of estimating the energy of a crystal is independent of the number of atoms in the crystal. The relative energies of different crystal phases of the same compound can be estimated using this method. Moreover, this fragmentation method can be applied to cleaved crystal surfaces and chemical reactions on such surfaces. ${ }^{20} \mathrm{~A}$ major advantage of this approach over other electronic methods for crystals ${ }^{21-27}$ is that any of the ab initio methods established for molecules can be easily applied to crystals. The major limitation of this approach is that it cannot be usefully applied to metals or other conducting materials, where the electronic wavefunctions are intrinsically delocalized.

\footnotetext{
a) Author to whom correspondence should be addressed. Electronic mail: michael.collins@anu.edu.au.
}

This paper improves the accuracy of the previously published fragmentation method in two ways: employing a more accurate distributed multipole description of the local electron density which improves the description of long range electrostatic interactions, and by including van der Waals or dispersion interactions at long range. Examples of the effect of these long range interactions on the crystal structure are presented. The paper also presents, for the first time, calculations of the harmonic lattice dynamics of crystals via systematic fragmentation. Comparison of results for phonon frequencies with neutron scattering experiments demonstrates the utility of the approach.

The paper is set out as follows. Section II presents a brief summary of the published method for systematic fragmentation of crystals and of how energies and other properties are calculated. Methods for accurately estimating long range electrostatic and dispersion interactions are also presented. Section III contains some details of the calculation of phonon frequencies and scattering intensities. Applications of the method to diamond, silicon, and $\alpha$-quartz are presented in Sec. IV to demonstrate the accuracy and utility of the approach. Some concluding remarks are contained in the final section.

\section{METHODS}

\section{A. Systematic fragmentation}

The fragmentation of molecules, crystals, and crystal surfaces has been described in detail previously, so only an abbreviated summary is included herein. Molecules and crystals are viewed as collections of functional groups connected by single bonds, where functional groups have the usual chemical meaning. In crystals, a functional group might often be an atom. Atoms which are formally connected by multiple bonds are contained in the same functional group. The fundamental step in fragmenting a structure is as follows: (i) break one bond to give a new structure (1); (ii) do not break this bond, but break another bond which is separated from the first by $N_{\text {Level }}$ functional groups to give a new structure (2); break both bonds in the original structure to give structure (3); add 
structures (1) and (2) and subtract structure (3) to give a composite structure. Repeat this process on all parts of the composite structure until there are no bonds separated by $N_{\text {Level }}$ functional groups. This approach is made systematic simply by carrying it out with $N_{\text {Level }}=1$ (called Level 1 fragmentation), then with $N_{\text {Level }}=2, N_{\text {Level }}=3$, and so on. A simple example is illustrative. A simple one-dimensional "crystal," based on a unit cell containing three functional groups, ABC, is represented by

$$
C^{(1 D)} \equiv \ldots . \mathrm{A}_{n-1} \mathrm{~B}_{n-1} \mathrm{C}_{n-1} \mathrm{~A}_{n} \mathrm{~B}_{n} \mathrm{C}_{n} \mathrm{~A}_{n+1} \mathrm{~B}_{n+1} \mathrm{C}_{n+1} \ldots
$$

At Level 1, the crystal is fragmented into an infinite sequence of one and two functional groups:

$$
C_{\text {Level1 }}^{(1 D)} \rightarrow \sum_{n=-\infty}^{\infty}\left[\begin{array}{l}
\mathrm{B}_{n-1} \mathrm{C}_{n-1}+\mathrm{C}_{n-1} \mathrm{~A}_{n}+\mathrm{A}_{n} \mathrm{~B}_{n} \\
-\mathrm{C}_{n-1}-\mathrm{A}_{n}-\mathrm{B}_{n}
\end{array}\right] .
$$

At Level 2:

$$
C_{\text {Level2 }}^{(1 D)} \rightarrow \sum_{n=-\infty}^{\infty}\left[\begin{array}{l}
\mathrm{B}_{n-1} \mathrm{C}_{n-1} \mathrm{~A}_{n}+\mathrm{C}_{n-1} \mathrm{~A}_{n} \mathrm{~B}_{n}+\mathrm{A}_{n} \mathrm{~B}_{n} \mathrm{C}_{n} \\
-\mathrm{B}_{n-1} \mathrm{C}_{n-1}-\mathrm{C}_{n-1} \mathrm{~A}_{n}-\mathrm{A}_{n} \mathrm{~B}_{n}
\end{array}\right]
$$

The fragments, e.g., $\mathrm{C}_{n-1} \mathrm{~A}_{n}$, are "capped" with hydrogen atoms, as previously described. ${ }^{12,13}$ The total electronic energy of the one-dimensional array is approximated at Level 2 by

$$
\begin{aligned}
& E_{1 D}^{\text {(Level2) }} \\
& =\sum_{n=-\infty}^{\infty}\left[\begin{array}{l}
\mathrm{E}\left(\mathrm{B}_{n-1} \mathrm{C}_{n-1} \mathrm{~A}_{n}\right)+\mathrm{E}\left(\mathrm{C}_{n-1} \mathrm{~A}_{n} \mathrm{~B}_{n}\right)+\mathrm{E}\left(\mathrm{A}_{n} \mathrm{~B}_{n} \mathrm{C}_{n}\right) \\
-E\left(\mathrm{~B}_{n-1} \mathrm{C}_{n-1}\right)-E\left(\mathrm{C}_{n-1} \mathrm{~A}_{n}\right)-E\left(\mathrm{~A}_{n} \mathrm{~B}_{n}\right)
\end{array}\right],
\end{aligned}
$$

where, for example, $\mathrm{E}\left(\mathrm{B}_{n-1} \mathrm{C}_{n-1} \mathrm{~A}_{n}\right)$ denotes the electronic energy of the molecule $\mathrm{HBCAH}$. The geometry of the BCA fragment is the same as that in the crystal, and the $\mathrm{H}$ atom caps are positioned along the corresponding bonds in the crystal. Inspection of the terms inside the square brackets in Eqs. (2.3) and (2.4) shows that there is a net residual (after addition and subtraction) of one of each of the groups A, B, and C. The net energy in the square brackets in Eq. (2.4) is an estimate of the energy per unit cell. As the Level of fragmentation increases, this energy is evaluated with increasing account of the bonding environment of each group.

A computer algorithm can apply the Level K fragmentation procedures to any crystal structure in three dimensions, as previously reported. ${ }^{19}$ The position of any atom in a crystal is given by

$$
\mathbf{x}\left(i, l_{1}, l_{2}, l_{3}\right)=\mathbf{x}(i, 0,0,0)+l_{1} \mathbf{a}(1)+l_{2} \mathbf{a}(2)+l_{3} \mathbf{a}(3),
$$

where $i$ numbers the atoms in a unit cell, up to $N$, the $\{\mathbf{a}(\mathrm{j})\}$ represent the lattice vectors, and $\left(l_{1}, l_{2}, l_{3}\right)$ are integers that enumerate the unit cells in the crystal. When the crystal is fragmented, some fragments can be associated with the "central unit cell," for example, the terms in the square brackets of Eq. (2.3) with $n=0$. A fragment that is associated with the central unit cell is denoted by $F_{n}(0,0,0)$. A fragment that is related to this by a simple lattice translation is denoted by $F_{n}\left(k_{1}, k_{2}, k_{3}\right)$, and by definition if $\mathbf{x}\left(i, l_{1}, l_{2}, l_{3}\right)$ is contained in $F_{n}(0,0,0)$, then $\mathbf{x}\left(i, k_{1}+l_{1}, k_{2}+l_{2}, k_{3}+l_{3}\right)$ is contained in $F_{n}\left(k_{1}, k_{2}, k_{3}\right)$. Corresponding to Eq. (2.3), the fragmentation of a crystal lattice can be denoted by

$$
C \rightarrow \sum_{k_{1}=-\infty}^{\infty} \sum_{k_{2}=-\infty}^{\infty} \sum_{k_{3}=-\infty}^{\infty} \sum_{n=1}^{N_{\text {frag }}} c_{n} F_{n}\left(k_{1}, k_{2}, k_{3}\right),
$$

where the $\left\{c_{n}\right\}$ are simple integers. As examples, the supplementary material ${ }^{28}$ presents such fragmentations of $\alpha$-quartz at Levels 1 to 4 . The corresponding energy of the entire crystal lattice is then given by

$$
\begin{aligned}
E_{b}^{\text {crys }} & =\sum_{k_{1}=-\infty}^{\infty} \sum_{k_{2}=-\infty}^{\infty} \sum_{k_{3}=-\infty}^{\infty} \sum_{n=1}^{N_{\text {frag }}} c_{n} E_{n}[\{\mathbf{x}[m(i), \mathbf{l}(i)+\mathbf{k}], \\
i & \left.\left.=1, \ldots, N_{a}(n)\right\}\right],
\end{aligned}
$$

where $E_{n}$ is the electronic energy of the fragment $F_{n}, N_{a}(n)$ is the number of groups in the $n$th fragment, and $\mathbf{x}$ is the Cartesian vector defined in Eq. (2.5). We have denoted this energy with a subscript $b$, to indicate that this energy is associated with the bonded interactions between functional groups in the crystal. At Level 1, fragments only contain at most two bonded groups, and only the energy due to bonding between nearest neighbor groups is accounted for in Eq. (2.7). At Level 2, second nearest neighbor effects are accounted for, and so on.

Since, every unit cell in a crystal has the same composition and structure, the energy of the crystal per unit cell, due to bonded interactions, can be estimated as

$$
E_{b}^{\mathrm{UC}}=\sum_{n=1}^{N_{\text {frag }}} c_{n} E_{n}\left[\left\{\mathbf{x}[m(i), \mathbf{l}(i)], \quad i=1, \ldots, N_{a}(n)\right\}\right] .
$$

The energy of a crystal fragment is a function of the positions of all the atoms in that fragment. Of course, the energy is independent of the six Cartesian coordinates that specify the position and orientation of the fragment.

\section{Additional symmetry}

The number of fragments, $N_{\text {frag }}$, depends on the number of groups in the unit cell and on their bonded connectivity. For example, silicon has eight atoms (groups) in the conventional unit cell, all connected to four other atoms. At Level 2, $N_{\text {frag }}$ $=24$, consisting of eight $\mathrm{Si}\left(\mathrm{SiH}_{3}\right)_{4}$ and $16 \mathrm{Si}_{2} \mathrm{H}_{6}$ fragments. Because silicon has such high symmetry (all $\mathrm{Si}$ atoms lie at the center of equivalent tetrahedra), all eight $\mathrm{Si}\left(\mathrm{SiH}_{3}\right)_{4}$ have the same structure (and energy), and all $16 \mathrm{Si}_{2} \mathrm{H}_{6}$ fragments have the same structure (and energy). Hence, it is only necessary to evaluate the energies of one $\mathrm{Si}\left(\mathrm{SiH}_{3}\right)_{4}$ and one $\mathrm{Si}_{2} \mathrm{H}_{6}$ fragment in order to evaluate $E_{b}^{\mathrm{UC}}$ in Eq. (2.8). In practice, no two fragments have exactly the same structure, since the position of the atoms is not known to infinite precision. For example, the Cartesian positions may be obtained from experimental data only to five or six significant figures. If two fragments are sufficiently close in structure, their energies are equal to many significant figures, and only one energy calculation is required. However, if the difference between the 
two structures exceeds some tolerance, then separate energy and gradient evaluations are necessary. For example, in optimizing the geometry of a crystal, the symmetry of the initial crystal structure may be broken, and optimization should proceed without enforcing a false symmetry. The algorithm for matching structures and subsequent manipulation of their energies and energy derivatives is described in Appendix A.

\section{B. Nonbonded effects}

Equation (2.8) only accounts for the electronic energy associated with functional groups and the bonding between those groups. However, functional groups may be well separated in a crystal in terms of bonding, but be close enough in space that there is a non-negligible energy of interaction between them. These relatively long range interactions are evaluated using perturbation theory.

In a previous paper, ${ }^{19}$ these long range interactions were enumerated in terms of a many-body expansion. More recent work $^{14}$ on fragmented molecules has shown that a more efficient approach is provided by a so-called "Level $1 \leftrightarrow$ Level 1 " description. The case of crystals differs somewhat from that of molecules, so some details are presented herein. As an aid to understanding, we begin with a treatment of nonbonded interactions that is simpler than the one we actually employ. We could approximate a crystal as a sum of individual groups:

$$
C \rightarrow \sum_{k_{1}=-\infty}^{\infty} \sum_{k_{2}=-\infty}^{\infty} \sum_{k_{3}=-\infty}^{\infty} \sum_{n=1}^{N} G_{n}\left(k_{1}, k_{2}, k_{3}\right)
$$

The energy due to the pairwise interaction of these groups with one another would be denoted by

$$
\begin{aligned}
E_{\text {pairs }}= & \frac{1}{2} \sum_{k_{1}=-\infty}^{\infty} \sum_{k_{2}=-\infty}^{\infty} \sum_{k_{3}=-\infty}^{\infty} \sum_{n=1}^{N} \sum_{j_{1}=-\infty}^{\infty} \sum_{j_{2}=-\infty}^{\infty} \\
& \times \sum_{j_{3}=-\infty}^{\infty} \sum_{m=1}^{N} E\left[G_{n}\left(k_{1}, k_{2}, k_{3}\right) \leftrightarrow G_{m}\left(j_{1}, j_{2}, j_{3}\right)\right],
\end{aligned}
$$

where $E\left[G_{n}\left(k_{1}, k_{2}, k_{3}\right) \leftrightarrow G_{m}\left(j_{1}, j_{2}, j_{3}\right)\right]$ is the interaction energy between two groups:

$$
\begin{aligned}
& E\left[G_{n}\left(k_{1}, k_{2}, k_{3}\right) \leftrightarrow G_{m}\left(j_{1}, j_{2}, j_{3}\right)\right]=E\left[G_{n}\left(k_{1}, k_{2}, k_{3}\right),\right. \\
& \left.G_{m}\left(j_{1}, j_{2}, j_{3}\right)\right]-E\left[G_{n}\left(k_{1}, k_{2}, k_{3}\right)\right]-E\left[G_{m}\left(j_{1}, j_{2}, j_{3}\right)\right] .
\end{aligned}
$$

In Eq. (2.10), self-interactions, $G_{n}\left(k_{1}, k_{2}, k_{3}\right)$ $\leftrightarrow G_{n}\left(k_{1}, k_{2}, k_{3}\right)$, are excluded from the sum. Rather than amend this "two-body" expression to account for many-body effects, we note that the crystal can be expressed as a Level 1 fragmentation:

$$
C \rightarrow \sum_{k_{1}=-\infty}^{\infty} \sum_{k_{2}=-\infty}^{\infty} \sum_{k_{3}=-\infty}^{\infty} \sum_{n=1}^{N_{\text {frag }}} c_{n} F_{n}^{\text {(Level1) }}\left(k_{1}, k_{2}, k_{3}\right) .
$$

The interaction energy can then be expressed as

$$
\begin{aligned}
& E_{\text {Level1 } \leftrightarrow \text { Level1 }} \\
& =\frac{1}{2} \sum_{k_{1}=-\infty}^{\infty} \sum_{k_{2}=-\infty}^{\infty} \sum_{k_{3}=-\infty}^{\infty} \sum_{n=1}^{N_{\text {frag }}} \sum_{j_{1}=-\infty}^{\infty} \sum_{j_{2}=-\infty}^{\infty} \sum_{j_{3}=-\infty}^{\infty} \sum_{m=1}^{N_{\text {frag }}} \\
& \quad \times c_{n} c_{m} E\left[F_{n}^{(\text {Level1 })}\left(k_{1}, k_{2}, k_{3}\right) \leftrightarrow F_{m}^{(\text {Level1 })}\left(j_{1}, j_{2}, j_{3}\right)\right],
\end{aligned}
$$

where self-interactions of groups are also excluded. The Level 1 fragments contain one or two functional groups, so the interaction energy

$$
\begin{aligned}
E[ & \left.F_{n}^{(\text {Level1) }}\left(k_{1}, k_{2}, k_{3}\right) \leftrightarrow F_{m}^{(\text {Level1 })}\left(j_{1}, j_{2}, j_{3}\right)\right] \\
= & E\left[F_{n}^{(\text {Level1) }}\left(k_{1}, k_{2}, k_{3}\right), F_{m}^{(\text {Level1 })}\left(j_{1}, j_{2}, j_{3}\right)\right] \\
& \quad-E\left[F_{n}^{(\text {Level1) }}\left(k_{1}, k_{2}, k_{3}\right)\right]-E\left[F_{m}^{(\text {Level1 })}\left(j_{1}, j_{2}, j_{3}\right)\right]
\end{aligned}
$$

contains up to four-group interactions (thinking in terms of a many-body expansion). In principle, the nonbonded energy of Eq. (2.13) could be systematically improved by using higher Level fragmentations. However, calculations on molecules have shown that Eq. (2.13) is sufficiently accurate. ${ }^{14}$ Now, we are only concerned with evaluating the energy of a crystal per unit cell. Hence, we will evaluate

$$
\begin{aligned}
E_{\text {Level1 } \mathrm{UC} \text { Level1 }}^{\mathrm{UC}} & \frac{1}{2} \sum_{k_{1}=-\infty}^{\infty} \sum_{k_{2}=-\infty}^{\infty} \sum_{k_{3}=-\infty}^{\infty} \sum_{n=1}^{N_{\text {frag }}} \sum_{m=1}^{N_{\text {frag }}} c_{n} c_{m} E \\
& \times\left[F_{n}^{(\text {Level1 })}\left(k_{1}, k_{2}, k_{3}\right) \leftrightarrow F_{m}^{(\text {Level1) }}(0,0,0)\right] .
\end{aligned}
$$

That is, we evaluate the interaction energy of all Level 1 fragments in the "central unit cell" with all other fragments in the crystal. In practice, when the distance between fragments is sufficiently large, this interaction energy is negligibly small. Hence, the infinite sums in Eq. (2.15) in practice reduces to a sum over fragments within some moderately large distance from fragments in the central unit cell (20-50 $\mathrm{a}_{\mathrm{o}}$ in our calculations). This truncation of the sums is justified for nonferroelectric materials, where every unit cell has a net zero dipole moment as well as zero net charge. We restrict our attention herein to such nonferroelectric materials.

Now we have indicated that self-interactions are excluded in Eq. (2.15). In addition, the interaction energy of groups which occupy common fragments has already been accounted for in the bonded energy of Eq. (2.8). For example, if the bonded energy of Eq. (2.8) was evaluated for a Level 2 fragmentation, all interactions between nearest neighbor and second nearest neighbor groups has been evaluated. Hence, such interactions are excluded in Eq. (2.15). Details of how such interactions are excluded are given in Ref. 14 for the corresponding nonbonded interactions in molecules. Therefore, Eq. (2.15) describes the interaction of groups which are at least third nearest neighbors ( $\gamma$ substituents) in terms of bonding. Such groups are normally separated by more than a few angstrom. Hence, we can evaluate all the interaction energies in Eq. (2.15) using perturbation theory. ${ }^{29}$ The long 
range interaction energy between molecules can be decomposed into contributions from electrostatic, dispersion (Van der Waals), and induction effects. We have found, from studies on molecules and clusters, that induction effects due only to long range electrostatic interactions are very small and are neglected herein. However, long range induction effects might be more significant in ionic crystals, and might need to be incorporated in such cases. We evaluate the long range electrostatic interactions using ab initio calculations of the charge distributions of the Level 1 fragments in Eq. (2.15). These charge distributions are represented by multipole moments distributed on all the atoms in the fragments, calculated using Stone's GDMA program ${ }^{30}$ and the ab initio electron density at the level of $a b$ initio theory employed for the calculation of the crystal energy. Ab initio calculations of the static polarizability and imaginary frequency dependent polarizability of the functional groups are carried out to determine the dispersion interactions between groups at long range. The details of these calculations have been presented previously in the context of molecular energies. ${ }^{14}$ It is important to note that all these long range interaction calculations are based on ab initio calculations of molecular properties and contain no adjustable or empirical parameters.

Explicitly, the nonbonded energy per unit cell is given by

$$
E_{\mathrm{nb}}^{\mathrm{UC}}=E_{\mathrm{Level} 1 \leftrightarrow \text { Level1 }}^{\mathrm{UC}}(\text { electrostatic })+E_{\mathrm{dispersion}}^{\mathrm{UC}} .
$$

Here the electrostatic energy is the sum of interactions between fragments:

$$
\begin{aligned}
& E_{\text {Level1 } \mathrm{ULevel1}}^{\mathrm{UC}}(\text { electrostatic) } \\
& =\frac{1}{2} \sum_{k_{1}=-\infty}^{\infty} \sum_{k_{2}=-\infty}^{\infty} \sum_{k_{3}=-\infty}^{\infty} \sum_{n=1}^{N_{\text {frag }}} \sum_{m=1}^{N_{\text {frag }}} c_{n} c_{m} E_{\text {elect }} \\
& \times\left[F_{n}^{(\text {Level1) }}\left(k_{1}, k_{2}, k_{3}\right) \leftrightarrow F_{m}^{(\text {Level1) }}(0,0,0)\right] .
\end{aligned}
$$

The fragment interactions are sums of atomic interactions:

$$
\begin{gathered}
E_{\text {elect }}\left[F_{n}^{(\text {Level1) }}\left(k_{1}, k_{2}, k_{3}\right) \leftrightarrow F_{m}^{(\text {Level1) }}(0,0,0)\right] \\
=\sum_{i \in F_{n}^{\text {(Levell) }}} \sum_{j \in F_{m}^{\text {(Level1) }}} E_{\text {elect }}(i, j) .
\end{gathered}
$$

Each atom in each fragment has a charge, q, dipole moment, $\boldsymbol{\mu}$, and quadrupole moment, $\Theta$, as evaluated by the GDMA program. $E_{\text {elect }}(i, j)$ is given by the electrostatic interactions of these moments [see Eq. (3.2.1) in Ref. 29]. For convenience, we have used Cartesian multipole moments. The electrostatic interactions depend on powers of the inverse distance between the atoms, $T=\|\mathbf{x}(j)-\mathbf{x}(i)\|^{-1}$, and derivatives of this distance with respect to the interatomic position vector. From earlier studies of electrostatic interactions in molecules, it was determined that distributed moments higher than the quadrupole make a negligible contribution to the electrostatic interaction at the distances relevant to Eq. (2.17).

The dispersion interaction is calculated as a pairwise interaction between groups in the central unit cell with all other groups

$$
\begin{aligned}
E_{\text {dispersion }}^{\mathrm{UC}}= & \frac{1}{2} \sum_{k_{1}=-\infty}^{\infty} \sum_{k_{2}=-\infty}^{\infty} \sum_{k_{3}=-\infty}^{\infty} \sum_{n=1}^{N} \sum_{m=1}^{N} \\
& \times E_{\mathrm{disp}}\left[G_{n}\left(k_{1}, k_{2}, k_{3}\right), G_{m}(0,0,0)\right] .
\end{aligned}
$$

Again, in Eq. (2.19), self-interactions and interactions between groups that occupy common fragments in the bonded energy evaluation are excluded. Letting $\alpha\left(n, k_{1}, k_{2}, k_{3}\right)$ represent the Cartesian static polarizability tensor of group $G_{n}\left(k_{1}, k_{2}, k_{3}\right), \mathbf{X}\left(n, k_{1}, k_{2}, k_{3}\right)$ represent the average Cartesian position of all atoms in $G_{n}\left(k_{1}, k_{2}, k_{3}\right)$, and $T$ $=\left\|\mathbf{X}\left(n, k_{1}, k_{2}, k_{3}\right)-\mathbf{X}(m, 0,0,0)\right\|^{-1}$, then (in atomic units):

$$
\begin{aligned}
& E_{\mathrm{disp}}\left[G_{n}\left(k_{1}, k_{2}, k_{3}\right), G_{m}(0,0,0)\right] \\
& \quad=-T_{\alpha \beta} T_{\gamma \delta} \alpha_{\alpha \gamma}\left(n, k_{1}, k_{2}, k_{3}\right) \alpha_{\beta \delta}(m, 0,0,0)\left[2 \frac{P_{n} P_{m}}{P_{n}+P_{m}}\right],
\end{aligned}
$$

where repeated indices are summed. The $P_{n}$ factors are evaluated from the imaginary frequency polarizability for group $G_{n}$, as previously described. ${ }^{14}$ This factor is a scalar, and is almost independent of small changes in the structure of the group. The infinite sums in Eq. (2.19) are truncated by excluding interactions where the groups are separated by more than 20-50 $\mathrm{a}_{\mathrm{o}}$. Equation (2.20) retains the full anisotropy of the dispersion interaction. For isotropic polarizabilities, Eq. (2.20) reduces to the familiar " $\mathrm{R}^{-6}$ " Van der Waals interaction. At higher levels of fragmentation, these nonbonded interactions only occur between groups that are separated by large distances. A perturbation expansion for the interaction would not be valid at shorter distances where the electron densities of the groups might significantly overlap. Hence, nonbonded interactions are also excluded if the distance between atoms in Level 1 fragments or groups is too small; here taken to be 1.5 times the sum of the Van der Waals radii of the atoms.

The total crystal energy per unit cell is given by

$$
E^{\mathrm{UC}}=E_{b}^{\mathrm{UC}}+E_{\mathrm{Level} 1 \leftrightarrow \text { Level1 }}^{\mathrm{UC}}(\text { electrostatic })+E_{\text {dispersion }}^{\mathrm{UC}} .
$$

\section{Additional symmetry}

Because the groups within one unit cell may be nearly structurally equivalent, the set of Level 1 fragments may contain only a very few structurally distinct molecules. Continuing the silicon example above, there is only one unique $\mathrm{Si}_{2} \mathrm{H}_{6}$ and one $\mathrm{SiH}_{4}$ structure in the set of Level 1 fragments. Hence, the distributed multipole electrostatic moments, the static polarizability tensor, and the $P$ factor need only be evaluated for one or two distinct structures. The tensor quantities are then rotated to the orientation required for each individual fragment in the Level 1 set or the set of groups, as described in Appendix A. Thus, the evaluation of the nonbonded energies only requires ab initio calculations on a very few molecular structures. 


\section{Geometry optimization}

In order to determine the minimum energy structure for a crystal, we must evaluate the gradient of the crystal energy. It is also convenient to evaluate the second derivatives of the energy for geometry optimization, as these second derivatives will also be employed in the lattice dynamics calculations. Those details of these calculations which have been presented previously ${ }^{13,19}$ will be omitted here.

\section{The bonding energy}

Under periodic conditions, the gradient of the total energy with respect to the position of atom $j$ in unit cell $\mathbf{k}$ is independent of $\mathbf{k}$. Thus, it is sufficient to evaluate the gradient of the potential energy surface (PES) with respect to atoms in the unit cell denoted by $\mathbf{k}=(0,0,0)$.

$$
\begin{aligned}
\frac{\partial E_{b}^{\mathrm{crys}}}{\partial x_{\alpha}(j, \mathbf{0})}= & \sum_{\mathbf{k}=-\infty}^{\infty} \sum_{n=1}^{N_{\text {frag }}} \sum_{i=1}^{N_{a}(n)} \\
& \times c_{n} \frac{\partial E_{n}\left[\left\{\mathbf{x}[m(i), \mathbf{l}(i)+\mathbf{k}], i=1, \ldots, N_{a}(n)\right\}\right]}{\partial x_{\alpha}[m(i), \mathbf{l}(i)+\mathbf{k}]} \\
& \times \delta_{j, m(i)} \delta_{\mathbf{l}(i)+\mathbf{k}, \mathbf{0}},
\end{aligned}
$$

where $\delta$ denotes the Kronecker delta, and $\delta_{\mathbf{l}(i)+\mathbf{k}, \mathbf{0}}$ should be understood as a product of three Kronecker delta functions, one for each vector component. Since all $\mathbf{k}$ values are included in the sum in Eq. (2.17), the value $\mathbf{k}=-\mathbf{l}(i)$ is present in the sum, so that $\delta_{\mathbf{l}(i)}+k, 0=1$ for some value of $\mathbf{k}$. This means that there are contributions to the energy gradient $\partial E_{b}^{\text {crys }} / \partial x_{\alpha}(j, \mathbf{0})$ from every fragment in Eq. (2.8) which contains the $j$ th atom in some unit cell. Effectively, the energy gradients which are assigned to atoms in the central unit cell are gradients of the bonding energy per unit cell, $E_{b}^{\mathrm{UC}}$.

The capping hydrogen atoms, contained in each fragment, contribute to the energy gradient for the atoms in the bond which has been broken, since the position of the $\mathrm{H}$ atom is determined completely by the positions of the bonded atoms. The position of each $\mathrm{H}$ atom is taken to lie along the "missing" bond vector at a distance that is proportional to the expected ratio of bond lengths. That is

$$
\mathbf{x}(\mathrm{H})=\mathbf{x}(i)+\frac{\operatorname{rad}(i)+\operatorname{rad}(\mathrm{H})}{\operatorname{rad}(i)+\operatorname{rad}(j)}[\mathbf{x}(j)-\mathbf{x}(i)],
$$

where $\mathbf{x}(i)$ denotes the Cartesian position of the atom in the fragment, $\mathbf{x}(j)$ denotes the Cartesian position of the bonded atom not in the fragment, and $\operatorname{rad}(i)$ denotes the covalent radius of atom $i$. Thus, for each capping $\mathrm{H}$ atom contained in a fragment, the gradient of the fragment energy with respect to that cap is in fact a gradient of the lattice energy with respect to the two lattice atoms that define the position of the cap. However, each capping $\mathrm{H}$ atom appears in Eqs. (2.6) and (2.7) in fragments with coefficients, $c_{n}$, which sum to zero. Hence, the net contribution of the $\mathrm{H}$ atom caps to the total energy gradient is small. The near cancellation of the effects of the $\mathrm{H}$ atom caps on the energy is discussed in more detail in Ref. 12.
The energy of the crystal depends on the unit cell parameters (the lattice vectors) because the relative positions of the atoms outside the central unit cell depend on the lattice vectors, as shown in Eq. (2.5). The gradient of the crystal bonding energy per unit cell can be found from Eq. (2.8) from

$$
\begin{aligned}
\frac{\partial E_{b}^{\mathrm{UC}}}{\partial a_{\alpha}(v)} & =\sum_{i=1}^{\text {natom }} \frac{\partial E_{b}^{U C}}{\partial x_{\alpha}(i)} \frac{\partial x_{\alpha}(i)}{\partial a_{\alpha}(v)} \\
& =\sum_{i=1}^{\text {natom }} \frac{\partial E_{b}^{\mathrm{UC}}}{\partial x_{\alpha}(i)} l_{v}(i), \quad \alpha=1,2,3 ; v=1,2,3
\end{aligned}
$$

where natom is the number of atoms which occur in at least one fragment in Eq. (2.8). Again, the $\mathrm{H}$ atom caps contribute to the gradients with respect to the lattice parameters, since the $\mathrm{H}$ atom cap position depends on the positions of the originally bonded atoms, which depend on the lattice parameters.

\section{The nonbonded energy}

The gradient of the long range electrostatic energy with respect to atomic positions in the unit cell and with respect to the lattice parameters is determined, by the chain rule, by the gradients of the pairwise atom-atom interaction, $E_{\text {elect }}(i, j)$. An example of a multipole-multipole interaction contribution to $E_{\text {elect }}(i, j)$ would be $\mu_{\alpha}(i) T_{\alpha \beta \gamma} \Theta_{\beta \gamma}(j)$ where repeated indices are summed. There are three distinct contributions to the derivatives of such terms. First, the $T$ tensors depend simply on the relative distance between the atoms, and the derivatives of these tensors with respect to $\mathbf{x}(\mathbf{i})$ and $\mathbf{x}(\mathrm{j})$ are easily evaluated. Second, if an atom in one fragment is infinitesimally displaced, the orientation of that fragment is infinitesimally rotated. The dipole and quadrupole moments of all atoms in that fragment are correspondingly rotated. This infinitesimally changes the interaction energy. This type of contribution to the electrostatic energy has been calculated for a number of test cases. It has been found that this contribution to the energy gradient is 2 orders of magnitude smaller than that arising from the $T$ tensors. The computation time for this very small contribution is high, so all such effects have been neglected. The third contribution to the derivatives of Eq. (2.18) arises from the fact that if an atom in one fragment is infinitesimally displaced, the shape of the fragment is changed, and the distributed charges, dipoles, and quadrupole moments of all the atoms are infinitesimally changed. Evaluation of this contribution to the energy gradient would require evaluation of the derivatives of the charge distribution of a fragment with respect to its structure. This requires a very substantial computational effort for what might be expected to be an effect as small as that due to the infinitesimal rotations above. This third contribution is also neglected herein. As the examples below demonstrate, neglect of these very small contributions to the energy gradient do not cause noticeable problems in the geometry optimization, at least in these cases. 
The pairwise dispersion energy derivatives are given by (repeated indices are summed):

$$
\begin{aligned}
\frac{\partial E_{\text {disp }}\left[G_{n}\left(k_{1}, k_{2}, k_{3}\right), G_{m}(0,0,0)\right]}{\partial X_{\varepsilon}\left(n, k_{1}, k_{2}, k_{3}\right)} \\
=-\left[T_{\varepsilon \alpha \beta} T_{\gamma \delta}+T_{\alpha \beta} T_{\varepsilon \gamma \delta}\right] \alpha_{\alpha \gamma}\left(n, k_{1}, k_{2}, k_{3}\right) \alpha_{\beta \delta} \\
\quad \times(m, 0,0,0)\left[2 \frac{P_{n} P_{m}}{P_{n}+P_{m}}\right] .
\end{aligned}
$$

\section{Hessians}

The second derivatives of the bonded energy with respect to the positions of the atoms and the lattice parameters are evaluated by chain rule in a similar manner to Eqs. (2.22) and (2.24). This approach also provides the mixed derivatives

$$
\begin{aligned}
& \frac{\partial^{2} E_{b}^{\mathrm{UC}}}{\partial x_{\beta}(j, \mathbf{0}) \partial a_{\alpha}(v)}=\sum_{\mathbf{k}} \sum_{i=1}^{\text {natom }} \frac{\partial^{2} E_{b}^{U C}}{\partial x_{\beta}(j, \mathbf{k}) \partial x_{\alpha}(i)} l_{v}(i), \\
& \alpha=1,2,3 ; \beta=1,2,3 ; v=1,2,3,
\end{aligned}
$$

where $\mathbf{x}(j, \mathbf{k})$ is the position of any $j$ atom in the fragments of Eq. (2.24).

\section{Additional symmetry}

As noted above, many fragments that are required to evaluate the bonding energy are equivalent in structure for some crystals. In such cases only one instance of the equivalent structures need have the energy evaluated ab initio. Similarly, the energy gradients in Eq. (2.22) need only be evaluated for one instance of equivalent structures. The gradients for another equivalent structure are obtained by rotation of the $a b$ initio calculated gradient, with reassignment of the atomic gradients to the appropriate atom in the other structure. A computer algorithm, described in Appendix A, is used to examine all fragments to determine which structures are unique and which are relabeled, rotated copies. After ab initio evaluation of the gradients (and second derivatives) of the unique structures, another algorithm permutes and rotates the gradients (and hessians) to match the relabeled, rotated copies.

\section{Fractional coordinates}

The first and second derivatives of the energy with respect to all $x_{\beta}(j, \mathbf{0})$ and $a_{\alpha}(v)$ are evaluated as above. In many crystal structures, the relative positions of atoms within the unit cell may be approximately constant during a geometry optimization, in the sense that their fractional coordinates are nearly constant. The fractional coordinates $\{z(j, l), j=1, \ldots, N ; l=1, \ldots, 3\}$ are related to the Cartesian positions of the atoms in the unit cell by

$$
\begin{aligned}
\mathbf{x}\left(i, l_{1}, l_{2}, l_{3}\right)= & z(i, 1) \mathbf{a}(1)+z(i, 2) \mathbf{a}(2)+z(i, 3) \mathbf{a}(3) \\
& +l_{1} \mathbf{a}(1)+l_{2} \mathbf{a}(2)+l_{3} \mathbf{a}(3) .
\end{aligned}
$$

The derivatives of the energy with respect to the $3 \mathrm{~N}$ Cartesian coordinates are therefore transformed into derivatives with respect to the $3 \mathrm{~N}$ fractional coordinates. The optimization of the crystal geometry with respect to the $3 \mathrm{~N}$ frac- tional coordinates and the nine lattice parameters is carried out as described in Appendix B.

\section{Phonon modes}

\section{Frequencies}

The formalism for the evaluation of the lattice vibrations is standard, with the relevant force constants obtained within the fragmentation approximation. In the limit of low amplitude motion of the atoms, we write the instantaneous position of each atom as

$$
\mathbf{x}\left(i, l_{1}, l_{2}, l_{3}, t\right)=\mathbf{x}\left(i, l_{1}, l_{2}, l_{3}\right)+\delta \mathbf{x}\left(i, l_{1}, l_{2}, l_{3}, t\right),
$$

where $\mathbf{x}\left(i, l_{1}, l_{2}, l_{3}\right)$ is given by Eq. (2.5). We define massweighted coordinates:

$$
y_{\alpha}\left(n, l_{1}, l_{2}, l_{3}, t\right)=\sqrt{m_{n}} \delta x_{\alpha}\left(n, l_{1}, l_{2}, l_{3}, t\right) .
$$

We define

$$
\begin{aligned}
& F_{\alpha \beta}\left(n, l_{1}, l_{2}, l_{3} ; m, j_{1}, j_{2}, j_{3}\right) \\
& \quad=\left.\frac{\partial^{2} E_{\text {crys }}}{\partial y_{\alpha}\left(n, l_{1}, l_{2}, l_{3}\right) \partial y_{\beta}\left(m, j_{1}, j_{2}, j_{3}\right)}\right|_{\mathbf{y}=0}
\end{aligned}
$$

and note that

$$
\begin{aligned}
& F_{\alpha \beta}\left(n, l_{1}, l_{2}, l_{3} ; m, j_{1}, j_{2}, j_{3}\right) \\
& \quad=F_{\alpha \beta}\left(n, 0,0,0 ; m, j_{1}-l_{1}, j_{2}-l_{2}, j_{3}-l_{3}\right), \\
& \quad=F_{\beta \alpha}\left(m, 0,0,0 ; n, l_{1}-j_{1}, l_{2}-j_{2}, l_{3}-j_{3}\right) .
\end{aligned}
$$

That is, because of the translational symmetry of the equilibrium lattice structure, the force constants only depend on the relative locations of the atoms. So, the equations of motion are

$$
\begin{aligned}
\frac{d^{2} y_{\alpha}\left(n, l_{1}, l_{2}, l_{3}, t\right)}{d t^{2}}= & -\sum_{m, j_{1}, j_{2}, j_{3}, \beta} F_{\alpha \beta}\left(n, l_{1}, l_{2}, l_{3} ; m, j_{1}, j_{2}, j_{3}\right) \\
& \times y_{\beta}\left(m, j_{1}, j_{2}, j_{3}, t\right) .
\end{aligned}
$$

We set

$y_{\alpha}\left(n, l_{1}, l_{2}, l_{3}, t\right)=u_{\alpha}(n ; \mathbf{q}, \omega) \exp \left[i\left(\mathbf{q} \cdot \mathbf{x}\left(n, l_{1}, l_{2}, l_{3}\right)-\omega t\right)\right]$,

where $\mathbf{q}$ is the phonon wavevector. Then a little standard algebra gives

$$
\begin{aligned}
\omega^{2} u_{\alpha}(n ; \mathbf{q}, \omega)= & \sum_{m, j_{1}, j_{2}, j_{3}, \beta} F_{\alpha \beta}\left(n, l_{1}, l_{2}, l_{3} ; m, j_{1}, j_{2}, j_{3}\right) \\
& \times u_{\beta}(m ; \mathbf{q}, \omega) \exp \left\{i \mathbf { q } \cdot \left[\mathbf{x}\left(m, j_{1}, j_{2}, j_{3}\right)\right.\right. \\
& \left.\left.-\mathbf{x}\left(n, l_{1}, l_{2}, l_{3}\right)\right]\right\} .
\end{aligned}
$$

This is a standard matrix eigenvalue problem for the phonon frequencies $\omega(\mathbf{q})$ and eigenvectors $\mathbf{u}(n ; \mathbf{q}, \omega)$. These eigenvectors are clearly complex, and in practice we solve the $6 \mathrm{~N}$ coupled equations for the real and imaginary parts of $\mathbf{u}(n ; \mathbf{q}, \omega)$. The harmonic force constants of Eq. (2.30) are calculated from the second derivatives of each term on the rhs of Eq. (2.21). The energy derivatives of the bonding energy 
arise from the energy derivatives of each fragment: each such derivative can be assigned to the complete Hessian via Eqs. (2.31a) and (2.31b), since we know which crystal atoms are contained in each fragment, or which crystal atoms defined each $\mathrm{H}$ atom cap in each fragment. For the long range electrostatic and dispersion energies, we can evaluate all the energy derivatives of Eqs. (2.17) and (2.19) which involve the central unit cell atoms, and assign these to the complete Hessian via Eqs. (2.31a) and (2.31b).

\section{Neutron scattering intensities}

The $6 \mathrm{~N} \times 6 \mathrm{~N}$ real symmetric matrix derived from Eq. (2.34) has $6 \mathrm{~N}$ real eigenvalues, but these are pairs of $3 \mathrm{~N}$ distinct values of $\omega^{2}(\mathbf{q})$. At a stable equilibrium all $3 \mathrm{~N}$ vibrational frequencies, $\omega(\mathbf{q})$, are real. These phonon mode frequencies can be measured in inelastic optical, $x$-ray, or neutron scattering. However, not all $3 \mathrm{~N}$ phonon modes have significant intensity in a particular scattering experiment. To assign observed peaks in a neutron scattering experiment (for example) to calculated phonon frequencies, it is useful to calculate the expected intensity of each peak. Here, the neutron scattering intensity for one phonon, $I_{\text {neut }}[j \mathbf{q}]$, is evaluated from the standard formula ${ }^{31}$ :

$$
\begin{aligned}
I_{\text {neut }}[j \mathbf{q}] \propto & \frac{1}{\omega_{j}(\mathbf{q})} \mid \sum_{\kappa=1}^{N}\left(m_{\kappa}\right)^{-1 / 2} b_{\kappa} \exp \left[-W_{\kappa}(\mathbf{Q})\right] \mathbf{Q} \cdot \mathbf{e}(\kappa \mid j \mathbf{q}) \\
& \times\left.\exp [i(\mathbf{Q}-\mathbf{q}) \mathbf{x}(\kappa, 0,0,0)]\right|^{2} \\
& \times \delta(\mathbf{Q}-\mathbf{q}-2 \pi \mathbf{H}),
\end{aligned}
$$

where the sum is over all the atoms in the central unit cell, $m_{\kappa}$ is the atomic mass, $b_{\kappa}$ is the coherent neutron scattering amplitude for each atom, ${ }^{32,33} \mathbf{Q}$ is the neutron scattering vector, $W_{\kappa}(\mathbf{Q})$ is the Debye-Waller factor, ${ }^{31} \mathbf{H}$ is a Bragg vector in the lattice reciprocal space, and $\mathbf{e}(\kappa \mid j \mathbf{q})$ is the (complex) eigenvector for the $j$ th eigenvalue from Eq. (2.34). $\mathbf{Q}, \mathbf{H}$, and $\mathbf{q}$ are related by

$$
\mathbf{Q}=2 \pi \mathbf{H}+\mathbf{q} .
$$

In order to evaluate the rhs of Eq. (2.35) for a particular phonon mode, the experimental value of $\mathbf{Q}$ (or equivalently the Bragg vector $\mathbf{H}$ ) must be known. For each value of the phonon wavevector $\mathbf{q}$, there are $3 \mathrm{~N}$ phonons, $j=1, \ldots$, $3 N$, but often only a subset of these phonons has significant intensity, due largely to the $\mathbf{Q} \cdot \mathbf{e}(\kappa \mid j \mathbf{q})$ factor in Eq. (2.35). The Debye-Waller factor for each atom ${ }^{31}$ is evaluated by a Monte Carlo summation over the allowed $\mathbf{q}$ vectors in the Brillouin zone.

\section{RESULTS}

\section{A. Structure and energy}

The geometries of $\alpha$-quartz, silicon and diamond have been optimized using selected levels of fragmentation, with Hartree-Fock and Möller-Plesset perturbation (MP2) methods with various Dunning correlation-consistent basis sets.
Tables of results are included in the supplementary material. ${ }^{28}$ Note that the nine lattice parameters (the Cartesian components of the three lattice vectors) and the fractional coordinates of all atoms in the conventional unit cell are free parameters in the geometry optimization. However, the number of each type of element in the unit cell is fixed. Hence, the optimization does not explore alternative minima which might be found for unit cells containing more (or less) atoms. Moreover, the optimization was initiated at the experimental geometry in each case. The supplementary material ${ }^{28}$ also presents tables of the energies of the optimized lattices, relative to the corresponding gas phase atoms.

It is important to note that it is only possible to make a qualitative comparison between these theoretical and experimental structures since (i) the theoretical structures are minimum energy geometries and contain no account of thermal (or even zero-point) averaging; and (ii) the experimental structures represent thermal averages (including net thermal expansion).

\section{1. $\alpha$-quartz}

The geometry of $\alpha$-quartz was optimized at Levels 2 and 3 at the MP2 level of ab initio theory with the cc-pVDZ and aug-cc-pVDZ basis sets. There are no large qualitative changes in the bond lengths, bond angles, or dihedral angles in the structure, compared to the experimental values. However, there are many small quantitative differences between, for example, the MP2/aug-cc-pVDZ and experimental geometries: on average, Si..O bond lengths differ by 0.0046 $\AA$, bond angles differ by $1.7^{\circ}$, and dihedral angles differ by $1.1^{\circ}$. The matrix of second derivatives of the crystal energy with respect to the fractional coordinates and the lattice parameters (hessian) is positive semidefinite (six eigenvalues are near zero, the rest are positive) at the optimized geometry. However, the initial (experimental) geometry has some negative eigenvalues for the hessian at this level of theory. This implies that the experimental geometry is near to a local maximum energy configuration, albeit also very close to an energy minimum. Taking the long range interactions into account leads to a minimum that is further distorted from the experimental structure: The Si..O bondlengths differ by $0.007 \AA$, bond angles differ by $2.0^{\circ}$, and dihedral angles differ by $3.5^{\circ}$, on average.

Figure 1 presents the energy of the Level 3/MP2/aug-ccpVDZ optimized geometry, evaluated using the MP2, CCSD, and $\operatorname{CCSD}(\mathrm{T})$ methods at different Levels of fragmentation, including the long range electrostatic and dispersion energies. Note that the lattice energy appears to converge with respect to the Level of fragmentation. The energy difference between Levels 3 and 4, for all three $a b$ initio methods, is only about $5 \mathrm{~kJ} \mathrm{~mol}^{-1}$, and about $23 \mathrm{~kJ} \mathrm{~mol}^{-1}$ between Levels 2 and 3 . It is also important to note that the CCSD and CCSD(T) energies only differ by about $7 \mathrm{~kJ} \mathrm{~mol}^{-1}$ at the higher levels of theory, while the MP2 method gives lattice energies which are about $100 \mathrm{~kJ} \mathrm{~mol}^{-1}$ below the $\operatorname{CCSD}(\mathrm{T})$ values. The supplementary material ${ }^{28}$ shows that dispersion is more important than the electrostatic interaction at long range; at the optimum 


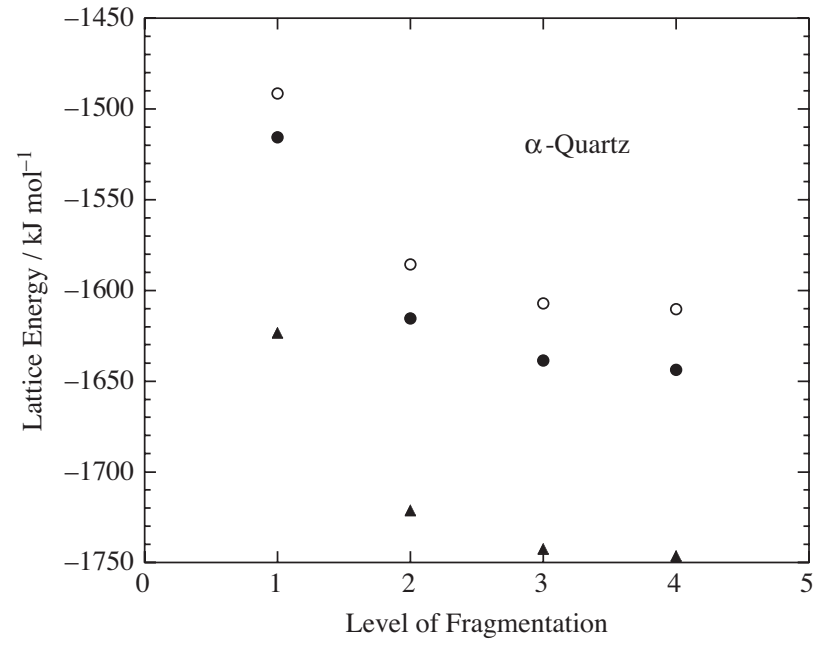

FIG. 1. The energy (in kJ per mole of $\mathrm{SiO}_{2}$ ) of the optimized $\alpha$-quartz lattice (not including the zero point energy) is shown as a function of the Level of fragmentation: $(\bullet) \operatorname{CCSD}(\mathrm{T}),(\mathrm{o}) \mathrm{CCSD},(\boldsymbol{\Lambda}) \mathrm{MP} 2$. The energy is given relative to that of $\mathrm{Si}+\mathrm{O}+\mathrm{O}$ in the gas phase.

geometry, the electrostatic interaction is about $1-2 \mathrm{~kJ}$ per mole of $\mathrm{SiO}_{2}$, while the dispersion energy is about -15 to -16 $\mathrm{kJ} \mathrm{mol}^{-1}$.

\section{Silicon}

For silicon, the supplementary material ${ }^{28}$ shows that the optimum Si..Si bond length varies slightly with level of $a b$ initio theory and level of fragmentation. It is not possible to fragment the lattice above Level 3 in this case. For Level 4, capping $\mathrm{H}$ atoms on different "ends" of a fragment would be very close. The "ring-repair rule," employed in molecular fragmentation to overcome this problem, ${ }^{13}$ would result in complete reconstitution of the whole lattice. The Si..Si bondlengths at Levels 2 and 3 are very similar. The long range forces associated with $E_{n b}$ are dominated by dispersion in this case (the electrostatic interaction is about 0 to $-1 \mathrm{~kJ}$ per mole of $\mathrm{Si}$, while the dispersion energy is about -5 to $\left.-8 \mathrm{~kJ} \mathrm{~mol}^{-1}\right)$. Not surprisingly then, incorporation of these attractive long range forces leads to a slight contraction of the Si..Si bondlength. For example, the Level 3/MP2/aug-ccpVDZ Si..Si bondlength of $2.3636 \AA$ contracts to $2.3543 \AA$ with account of the long range forces. The long range dispersion interactions lower the lattice energy by about $8 \mathrm{~kJ}$ per mole of silicon atoms.

The lattice energy of silicon (see supplementary material ${ }^{28}$ ) also varies substantially with the level of $a b$ initio theory. At Level 3, the CCSD(T) lattice energy is about 26 $\mathrm{kJ} \mathrm{mol}^{-1}$ above the corresponding MP2 value. The $\operatorname{CCSD}(\mathrm{T})$ energy falls by about $34 \mathrm{~kJ} \mathrm{~mol}^{-1}$ from Level 2 to Level 3; larger than the corresponding value of $23 \mathrm{~kJ} \mathrm{~mol}^{-1}$ for $\alpha$ quartz. Hence, although the lattice energy of $\alpha$-quartz appears to be reasonably converged by Level 3 fragmentation, the same may not be true for silicon.

\section{Diamond}

For diamond, it is not possible to fragment the lattice above Level 2. Here, capping H atoms on different "ends" of a fragment would be very close for both Levels 3 and 4, and the ring-repair rule reconstitutes the whole lattice. The supplementary material ${ }^{28}$ shows that the optimum C..C bond length varies slightly with the level of ab initio theory. The long range forces associated with $E_{n b}$ are again dominated by dispersion. Thus, incorporation of these attractive long range forces leads to a contraction of the C..C bondlength. For example, the Level 2 MP2/aug-cc-pVDZ C..C bondlength of $1.5447 \AA$ contracts to $1.5339 \AA$ with account of the long range forces. The long range dispersion energy is large, about -17 $\mathrm{kJ} \mathrm{mol}^{-1}$, compared to $-8 \mathrm{~kJ} \mathrm{~mol}^{-1}$ for silicon. Although the static polarizability of a silicon atom is about three times larger than that of a carbon atom, the imaginary frequency polarizability for carbon is larger than the corresponding silicon response, and most importantly, the shorter C..C bondlength ensures that nonbonded C....C interactions occur at shorter distances than for the corresponding Si....Si interactions. The total lattice energy of diamond (see supplementary material ${ }^{28}$ ) also varies substantially with the level of ab initio theory. At Level 2, the $\operatorname{CCSD}(\mathrm{T})$ lattice energy is about $43 \mathrm{~kJ} \mathrm{~mol}^{-1}$ above the corresponding MP2 value.

\section{B. Phonon frequencies}

Phonon frequencies were evaluated for a range of phonon wavevectors, using the positions of all atoms in the conventional unit cell in Eq. (2.34). The intensity associated with each frequency was evaluated using Eq. (2.35). The figures below present only those phonon frequencies with associated intensity above a given minimum [intensities were evaluated using the reciprocal lattice point $\mathbf{H}=(10 \overline{1})$ or (100) in Eq. (2.36)].

\section{Silicon}

Phonon frequencies were evaluated for silicon at the optimized geometries for each level of $a b$ initio theory employed. Figure 2 presents a comparison of frequencies determined by inelastic neutron scattering ${ }^{34,35}$ with those calculated at Level 3 fragmentation, using the MP2/aug-cc-pVDZ method with account of long range electrostatics and dispersion. The calculated frequencies in Fig. 2 are characteristic of the values obtained at fragmentation Levels 2 and 3 with all basis sets, with or without long range interactions. The supplementary information ${ }^{28}$ shows tables of the average variation of all phonon frequencies with variation of the ab initio and fragmentation methods. These data show that the most significant variation of the frequencies is produced by changing the level of fragmentation; the frequencies at Level 3 differ from those at Level 2 on average by about $0.4 \mathrm{THz}\left(13 \mathrm{~cm}^{-1}\right)$. Figure 3 illustrates the improved agreement between calculation and experiment that is obtained by increasing the fragmentation Level from 2 to 3 . The long range interactions also change the frequencies; on average by $0.18 \mathrm{THz}\left(6 \mathrm{~cm}^{-1}\right)$ for Level 2 , and by $0.15\left(5 \mathrm{~cm}^{-1}\right)$ for Level 3 . The data suggest that a major part of the frequency shift due to long range interactions is due to the associated change in optimized geometry. We recall that the attractive long range dispersion interaction leads 

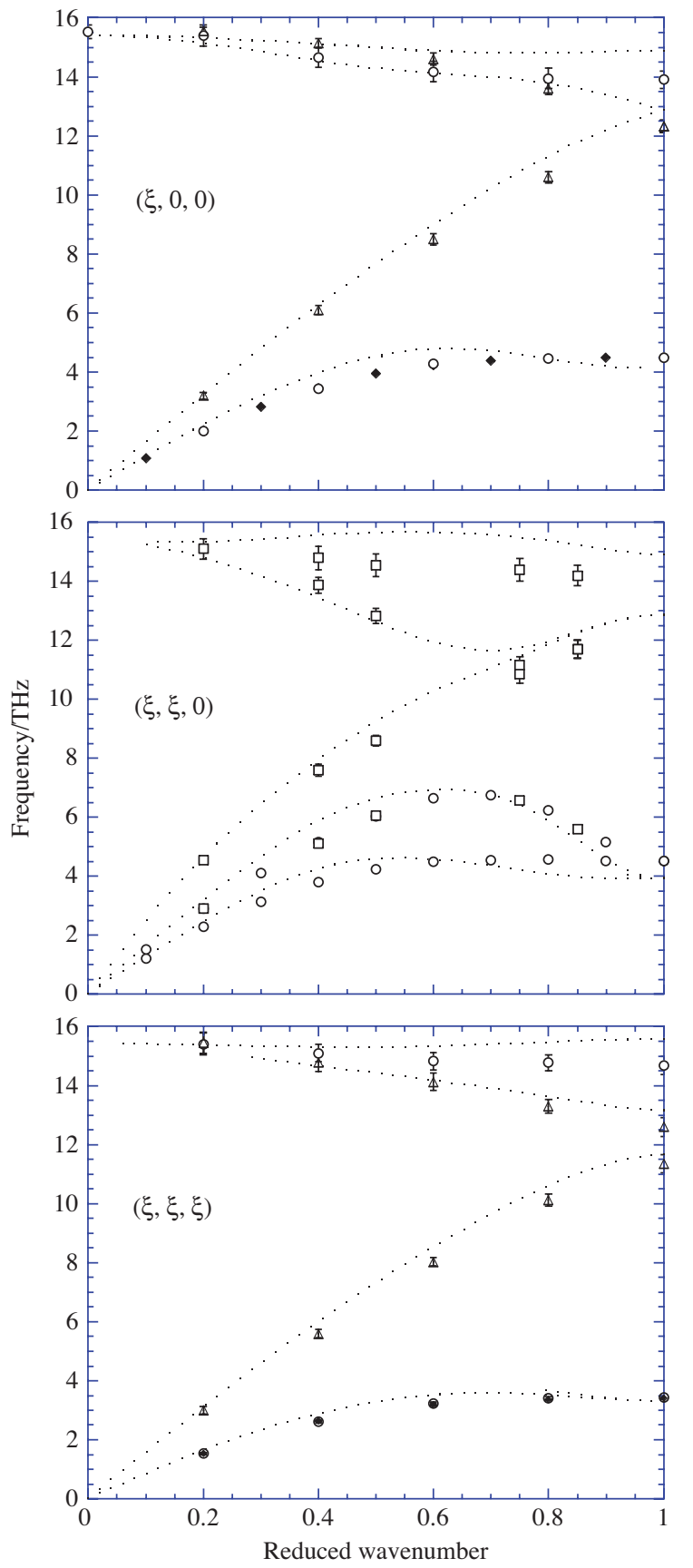

FIG. 2. Frequencies for silicon are shown for phonon wavevectors, $\mathbf{q}$, along the [100], [110], and [111] directions with respect to the axes of the conventional unit cell. The reduced wavenumber is defined as $|\mathbf{q}|$ divided by a maximum value: For [100] and [111] this maximum is the value of $|\mathbf{q}|$ at the Brillouin zone edge, while for [110], it is $4 / 3$ of the value of $|q|$ at the Brillouin zone edge. The dotted lines are the calculated values (Level 3/MP2/aug-cc$\left.\mathrm{pVDZ}+E_{n b}\right)$ and the symbols with error bars denote the experimental values from Refs. 34 and 35.

to a contraction of the equilibrium bond lengths. The second derivatives of the bonding energy, $E_{b}$, are different at this contracted geometry. Using a Lennard-Jones $(12,6)$ potential as a simple model, a little algebra shows that an addition to the long range attractive component contracts the equilibrium bond length, and produces an increase in the equilibrium second derivative due to the repulsive part of the potential.

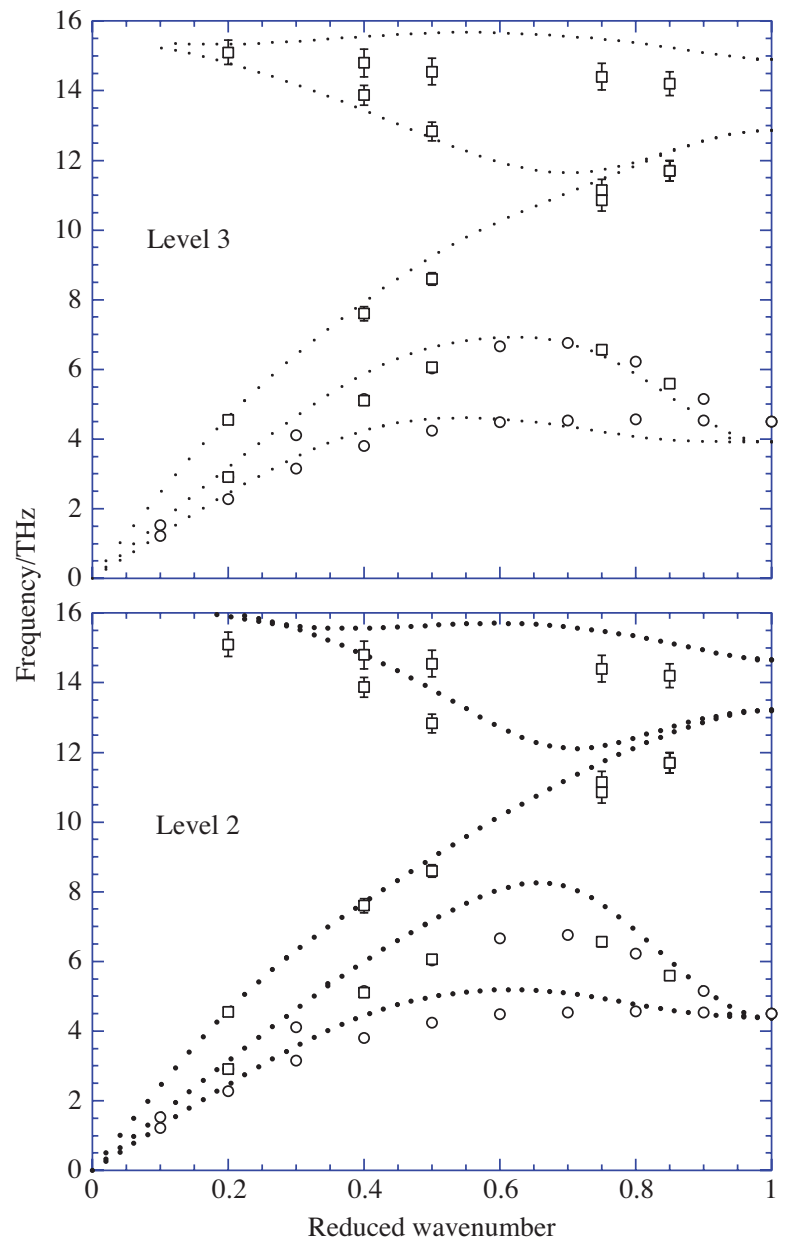

FIG. 3. Frequencies for silicon are shown along [110] (see Fig. 2), calculated at Levels 2 and 3 at MP2/aug-cc-pVDZ using $E_{b}+E_{n b}$.

Figure 2 clearly indicates that ab initio evaluation of the lattice vibrational frequencies can produce quantitative agreement with experimental data. It is important to note that these are the results of a "first-principles" calculation with no adjustable parameters.

\section{Diamond}

Figure 4 presents a comparison of frequencies determined by inelastic neutron scattering ${ }^{36,37}$ with those calculated at Level 2 fragmentation, using the MP2/cc-pVTZ method.

Again, these results are qualitatively characteristic of the values obtained using the MP2 method with all basis sets, with or without long range interactions (see the supplementary material ${ }^{28}$ ). An example of the effect of the long range interactions is presented in Fig. 5. The variation of the frequencies due to long range interactions is larger in magnitude than in silicon, albeit that the frequencies are larger in diamond by a factor of about 2-3 on average. Across all basis sets and phonon wavevectors, the mean variation in the phonon frequencies caused by long range interactions is about $0.53 \mathrm{THz}$ $\left(18 \mathrm{~cm}^{-1}\right)$, compared to $0.13 \mathrm{THz}$ at Level 2 for silicon. The principal cause of these changes in the phonon frequencies is 

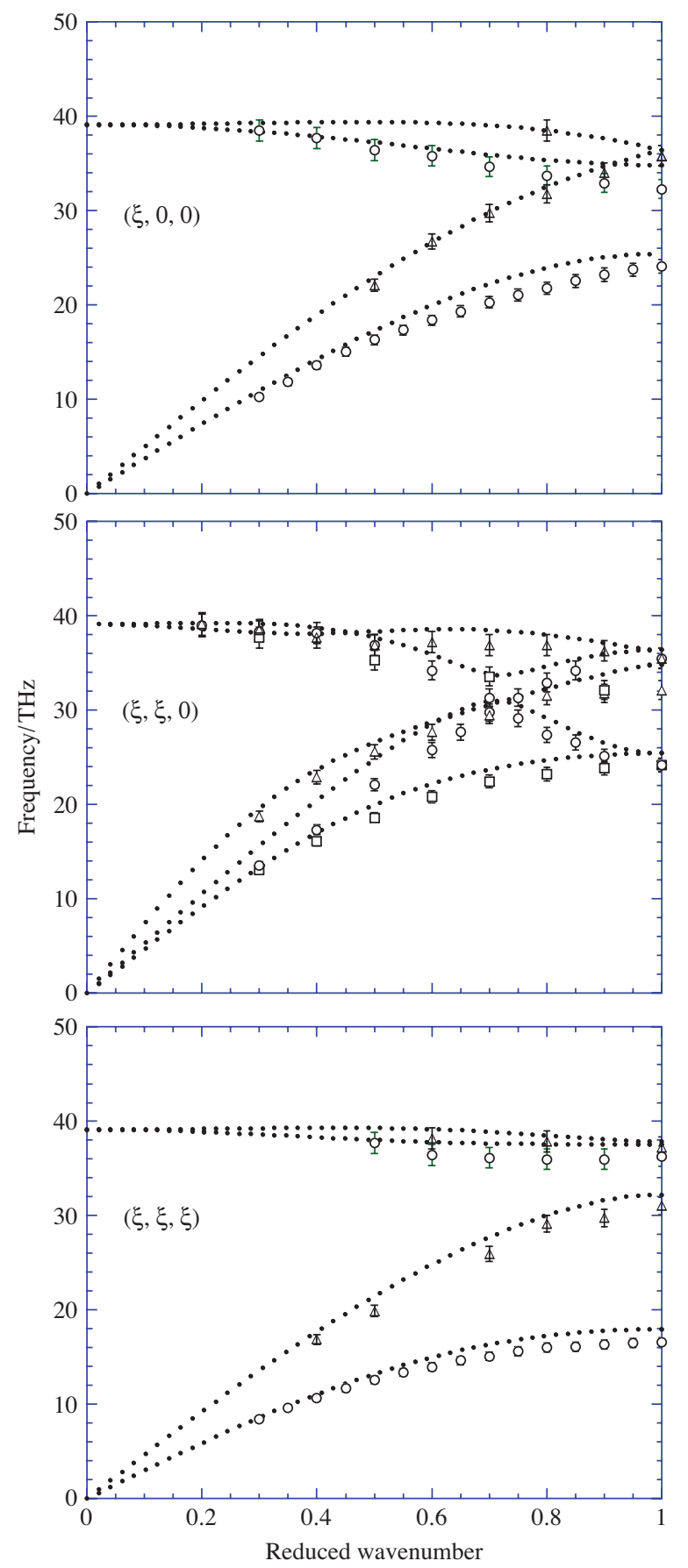

FIG. 4. Frequencies for diamond are shown for phonon wavevectors, $\mathbf{q}$, along the [100], [110], and [111] directions with respect to the axes of the conventional unit cell. The reduced wavenumber is defined as $|\mathbf{q}|$ divided by a maximum value: For [100] and [111] this maximum is the value of $|\mathbf{q}|$ at the Brillouin zone edge, while for [110], it is $4 / 3$ of the value of $|q|$ at the Brillouin zone edge. The dotted lines are the calculated values (Level 2/MP2/cc-pVTZ) and the symbols with error bars denote the experimental values from Refs. 36 and 37.

due to the change in the lattice constant caused by the relatively large long range dispersion interactions (see above). The agreement between calculation and experiment evident in Fig. 4 has been achieved using only Level 2 fragmentation. This accounts for an $a b$ initio estimate of the first and second nearest neighbor interactions.

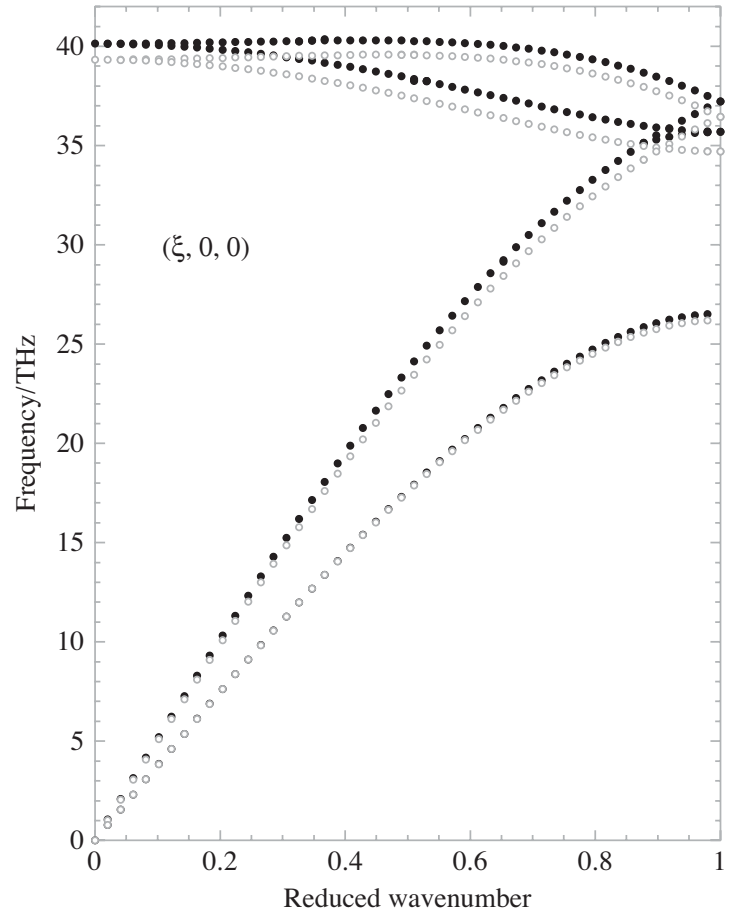

FIG. 5. Frequencies for diamond are shown for phonon wavevectors, $\mathbf{q}$, along the [100] direction as in Fig. 4. The frequencies have been evaluated with Level 2 fragmentation at the MP2/aug-cc-pVDZ level of theory with $(\bullet)$ and without $(\circ)$ the long range nonbonded interactions.

\section{3. $\alpha$-quartz}

Phonon frequencies were evaluated for $\alpha$-quartz at the optimized geometries for fragmentation Levels 2 and 3, using the MP2 method with the cc-pVDZ and aug-cc-pVDZ basis sets. Figure 6 presents a comparison of the experimental data ${ }^{38}$ (for phonons along the [001] direction in the conventional unit cell) with the calculated values for Level 3/MP2/aug-cc-pVDZ. All calculated phonon frequencies are shown. Figure 7 presents the corresponding data where the nonbonded interactions have been taken into account.

Although there is substantial qualitative and quantitative agreement between the experimental and calculated values, there are also significant differences. In the low frequency region, there is generally good agreement between theory and experiment. Note that the inclusion of the long range interactions improves the agreement between calculation and experiment for the lowest frequency acoustic mode. The supplementary material ${ }^{28}$ tabulates the variation of the phonon frequencies with basis set, level of fragmentation, and account of long range forces. The most notable difference between theory and experiment in Figs. 6 and 7 is seen for the highest energy optical modes, where theory consistently underestimates the frequencies.

There are a number of possible causes of this disagreement for the high frequency modes, including: a larger basis set and/or a higher level of $a b$ initio theory are necessary, and that the harmonic approximation for the frequencies is inadequate. To explore the effect of much larger basis sets and alternative levels of $a b$ intio theory on these frequencies, we have considered a model compound, $\left(\mathrm{SiH}_{3}\right) \mathrm{O}\left(\mathrm{SiH}_{3}\right)$. It was 

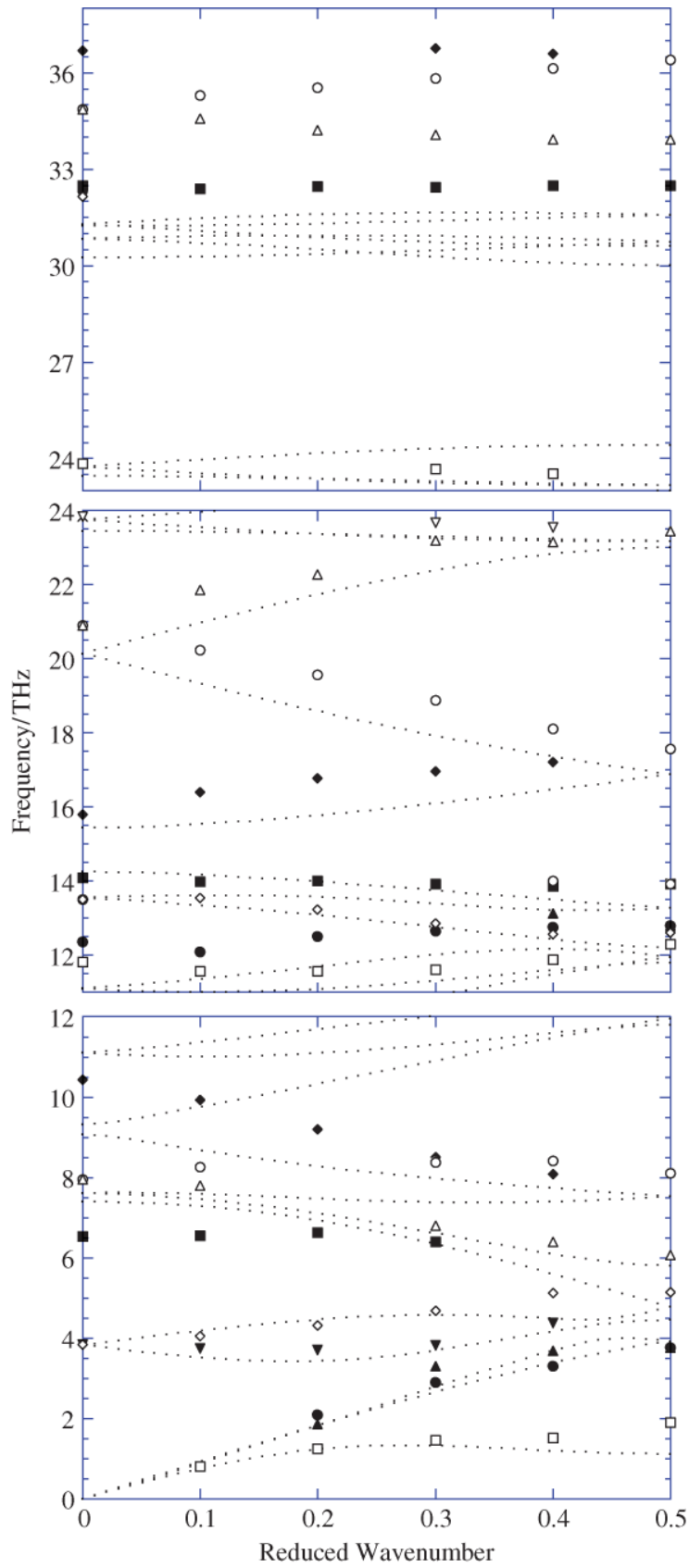

FIG. 6. Frequencies for $\alpha$-quartz are shown for phonon wavevectors, $\mathbf{q}$, along the [001] direction with respect to the axes of the conventional unit cell. The reduced wavenumber is $|\mathbf{q}|$ divided by the value of $|\mathbf{q}|$ at the Brillouin zone edge. The dotted lines are the calculated values (using $E_{b}$ only), for Level 3/MP2/aug-cc-pVDZ, and the symbols denote the experimental values from Ref. 38.

found that other ab initio methods, using basis sets up to augcc-pVTZ, do not give SiOSi stretching frequencies higher in value than MP2/aug-cc-pVDZ, which suggests that more reliable $a b$ initio methods would not raise the calculated phonon frequencies. Although the masses for oxygen and silicon are relatively large, anharmonic effects on the phonon frequencies may be significant. As noted above, the experimental crystal structure appears to be near a local energy maximum at MP2/aug-cc-pVDZ, although also very close to the minimum energy structure. The supplementary material ${ }^{28}$ also shows that the inclusion of long range forces further distorts the bond
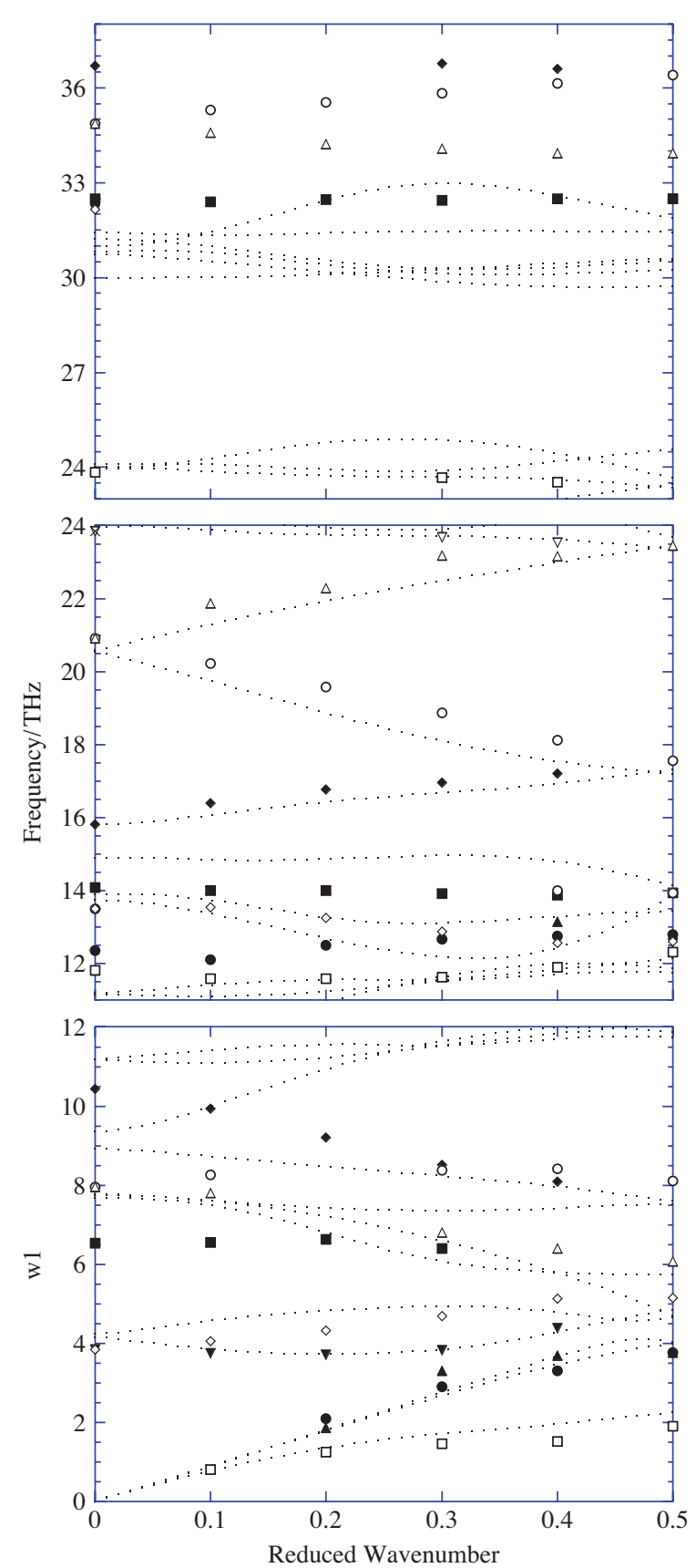

FIG. 7. The corresponding data to Fig. 6, except that the calculated values are obtained using $E_{b}+E_{n b}$ at Level 3/MP2/aug-cc-pVDZ.

lengths, angles, and dihedrals from the experimental values. Hence, it would appear that the energy surface for this crystal features low energy maxima near energy minima, and that the minimum energy structure is easily distorted by the addition of small forces. Hence, a simple harmonic model of the PES, near the minimum, may not be very accurate. A complete anharmonic description of the PES is beyond the scope of this paper.

\section{DISCUSSION}

The method of systematic fragmentation for the calculation of phonon frequencies has been presented and applied 
to three quite different cases. Although silicon and diamond have the same structure, they have very different electronic structures (one is an insulator, the other a semiconductor) and different lattice dynamics. $\alpha$-quartz has a much more open crystal structure, lower symmetry, and more complicated phonon spectrum. The quality of all the results indicates the promise of this approach generally.

There are limitations on the application of this approach. When the electronic structure is "delocalized," as in a metal, systematic fragmentation is unlikely to be accurate. From our studies of molecular energies, Level 1 fragmentation is not normally or reliably accurate enough to answer chemical questions. Figure 1 implies that Level 1 fragmentation is also not likely to be an accurate description for crystal energetics. For molecules, Level 2 fragmentation can often be accurate enough to provide useful estimates of chemical properties, and Fig. 4 indicates that Level 2 can be useful for estimating phonon frequencies. Systematic fragmentation is likely to be most useful for those crystal structures which are "open" in character, that is, not dominated by small rings. In such cases, the $\mathrm{H}$ atom caps do not spuriously interact with one another, and a sufficiently long sequence of Levels of fragmentation can be employed to verify convergence and accuracy (as exemplified by the case of $\alpha$-quartz). Molecular crystals are likely to be amenable to systematic fragmentation since no $\mathrm{H}$ atom caps are required to restore the valency when weak intermolecular "bonds" are broken.

A major advantage of applying systematic fragmentation to crystals is that reliable, high levels, of $a b$ initio theory can be employed. This means that a hierarchy of methods and basis sets can be used to determine convergence of energies and properties. For example, the supplementary material ${ }^{28}$ indicates that diffuse basis functions have a significant impact on estimates of the total lattice energy in the cases studied herein. The treatment of long range effects has been improved here, relative to earlier work, ${ }^{19}$ by the incorporation of dispersion and distributed-multipole electrostatics, evaluated within a Level 1 - Level 1 description of nonbonded interactions. This treatment has been shown to improve the accuracy of estimation of molecular energies for a substantial number of molecules. ${ }^{14}$ Hence, one can use high levels of ab initio theory combined with better estimates of long range interactions to evaluate lattice energies more accurately. We have demonstrated that this approach gives an apparently convergent estimate of the lattice energy for $\alpha$-quartz. The approach can be just as easily applied to many different crystals and to polymorphs of the same material, as in an earlier study of several polymorphs of silica. ${ }^{19}$ The method is not limited by the symmetry, composition, or size of the crystal unit cell. Hence, the relative lattice energies of different crystal phases can be estimated. The evaluation of phonon frequencies allows at least an estimate of relative free energies of different crystal phases.

The application of accurate molecular quantum chemistry methods to crystals has also recently been pursued by different methods, which are related to the systematic fragmentation presented herein. Sode et al. ${ }^{39}$ have applied a pairwise interaction approach to a chain model of crystalline hydrogen fluoride, taking account of the charge distribution of the crystal environment. They also included anharmonic ef- fects in their calculation of phonon frequencies. As noted above, the expansion of a lattice energy in terms of separate localized interactions is particularly appropriate for molecular crystals. Beran and Nanda ${ }^{40}$ have recently shown that the lattice energies of some molecular crystals could be obtained accurately using MP2 calculations of pairwise interactions at short range with long range interactions treated with polarizable molecular mechanics force fields. The recent method, which is closest in spirit to systematic fragmentation, is the use of a hierarchy of fragments or clusters. Nolan et al. ${ }^{41}$ have developed this approach with application to $\mathrm{LiH}$, which has the rock salt structure. All the above methods only require calculation and combination of energies, rather than wavefunctions. The "method of increments" spirit to fragment molecular orbital theory ${ }^{4,5}$ in using a localized representation of orbitals to construct the correlation energy of crystals. All these methods stand in contrast to density functional theory (DFT), which is the dominant paradigm for the electronic structure of crystals and lattice dynamics. ${ }^{27}$ The usual argument against the use of DFT is that it is not amenable to systematic improvement, and so it's accuracy cannot be independently estimated. Moreover, the results of a DFT calculation depend critically on the functional and any pseudopotential employed. This type of method cannot be truly a "first principles" approach. However, it remains to be seen if alternative methods, such as systematic fragmentation combined with high level ab initio methods, can surpass DFT in terms of accuracy and utility.

The estimation of long range interactions may require further improvement. For silicon and diamond it is not surprising that dispersion is the dominant long range interaction, since the $\mathrm{Si}$ and $\mathrm{C}$ atoms have no charge or dipole moment. For $\alpha$-quartz, the $\mathrm{Si}$ and $\mathrm{O}$ atoms are charged, but the unit cell as a whole has no net charge or net dipole moment (only ferroelectric materials have a net dipole moment for the unit cell). Here the long range electrostatic interaction is not negligible, though still much smaller than the dispersion interaction. Of course, it may be that the long range electrostatic interaction dominates long range dispersion for other crystals. If the unit cell has a net quadrupole moment, then the long range quadrupole-quadrupole interaction decays more slowly $\left(\mathrm{R}^{-5}\right)$ with distance than the dispersion interaction. If the long range electrostatic interaction is significant, it is then likely that induction due to the associated electric field should also be taken into account. We have routinely included this long range induction in calculations of molecular energies, ${ }^{14}$ but have neglected it herein as it has appeared to be very small. For ferro-electric materials, the dominant long range electrostatic interaction would be due to dipole-dipole interactions, with vary with distance as $\mathrm{R}^{-3}$. The sum of these interactions between one unit cell and all other cells does not converge, and one has to take the boundary conditions into account. It is likely then that the effect of long range induction would also have to be evaluated for such materials. Hence, if long range electrostatic interactions are large, the treatment of long range induction (already used for molecules) would need to be implemented for crystals. The previous investigation of induction effects and the treatment of dispersion herein have used polarizabilities for whole groups. In the event that such 
groups are large, a more accurate description might be obtained by distributing the polarizability to each atom in the group, in similar fashion to the way that electrostatic moments are distributed. Further investigations of a wide range of crystal structures, with a range of electronic structures, are required to determine the extent to which our treatment of long range interactions requires further improvement.

\section{ACKNOWLEDGMENTS}

The author gratefully acknowledges helpful discussion with his colleagues, Professors John White, Richard Welberry, and Ray Withers, and Dr. Terry Frankcombe.

\section{APPENDIX A: COMPARISON OF MOLECULAR SHAPES}

The task is to determine whether or not a molecular configuration, $\mathrm{C} 1$, has essentially the same shape as another configuration, $\mathrm{C} 2$, albeit that the order of the atoms and the orientation of the two configurations may be different. If the two configurations are essentially equivalent, then the data required to transform one configuration into the other must be evaluated. The algorithm to achieve this is as follows:

(1) Evaluate the centroid position of the atoms in C1, and subtract this from the coordinates of each atom to give coordinates $\{x l(n, i), n=1, \ldots, \mathrm{N}, i=1, ., 3\}$ with common center at the origin.

(2) Form a second rank tensor $(3 \times 3$ matrix $) \mathbf{M}$ :

$$
M_{i j}=\sum_{n \in C 1} m(n) x 1(n, i) x 1(n, j),
$$

where $m(n)$ denotes a "mass" for atom $n$, set to 1 for all but two atoms, for which $m$ takes an arbitrary value. We allocate these arbitrary masses to each possible pair of atoms, in turn, until $\mathbf{M}$ has three nondegenerate eigenvalues, $\{\mathrm{E} 1\}$. The corresponding eigenvectors, $\{\mathrm{V} 1\}$, then determine the orientation of $\mathrm{C} 1$.

(3) Repeat steps (1) and (2) for configuration C2, with coordinates $\{x 2\}$.

(4) Compare the eigenvalues $\{\mathrm{E} 1\}$ and $\{\mathrm{E} 2\}$. If $\{\mathrm{E} 1\}$ $\neq\{\mathrm{E} 2\}$, return to step (3) to obtain a new set of nondegenerate eigenvalues for a different allocation of the arbitrary masses. If $\{\mathrm{E} 1\} \neq\{\mathrm{E} 2\}$, for all possible allocation of the arbitrary masses, then $\mathrm{C} 1$ cannot be equivalent to $\mathrm{C} 2$ and we are finished. If $\{\mathrm{E} 1\}=\{\mathrm{E} 2\}$ then

(5) The origin and $\{\mathrm{V} 1\}$ define four points that define an irregular shape. The origin and $\{\mathrm{V} 2\}$ define also four points that define an irregular shape. The unitary matrix, A, which rotates the latter four points to be as close as possible to the first four points is evaluated (see for example, the Appendix in Ref. 45). The signs of the eigenvectors $\{\mathrm{V} 2\}$ are arbitrary, so that there are eight possible shapes defined by the origin and $\{\mathrm{V} 2\}$. Each of these eight possibilities are rotated to be as close as possible to the origin and $\{\mathrm{V} 1\}$. The choice of signs which results in the minimum distance is taken to correspond to the optimal matrix A.
(6) Perform the unitary transformation (a proper or an improper rotation) on the coordinates of $\mathrm{C} 2$ to give new coordinates $\mathbf{x}^{(2) \prime}=\mathbf{A} \mathbf{x}^{(2)}$.

(7) Find the atom number $m$ which makes $\mathbf{x}^{(2) \prime}(m)$ as close as possible to $\mathbf{x}^{(1)}(n)$ for all atoms $(n)$ in $\mathrm{C} 1$. This provides a table $\{\mathrm{Tbl}(\mathrm{n}, \mathrm{m})\}$ of the permutations of the numbering in $\mathrm{C} 1$ and $\mathrm{C} 2$. If the closest atom-atom distances exceed some tolerance, then $\mathrm{C} 2 \neq \mathrm{C} 1$, and we are finished. If all atom-atom distances are sufficiently small then $\mathrm{C} 2=\mathrm{C} 1$, and $\{\operatorname{Tbl}(n, m)\}$ and the matrix $\mathbf{A}$ provide all the necessary information to transform any tensorial properties of $\mathrm{C} 1$ to the corresponding properties of $\mathrm{C} 2$ (e.g., the energy gradient, dipole moment, or polarizability tensor).

\section{APPENDIX B: ENERGY MINIMISATION}

The method adopted to minimize the total lattice energy per unit cell, Eq. (2.21), is simple and robust, but computationally profligate. The first and second derivatives of the energy with respect to the $3 \mathrm{~N}$ atomic coordinates of the central unit cell and the nine lattice parameters are transformed, using Eq. (2.27) into the first, $\mathbf{g}$, and second derivatives, $\mathbf{F}$, of the energy with respect to the $3 \mathrm{~N}$ fractional coordinates and the nine lattice parameters. We denote the $(3 \mathrm{~N}+9) \times 1$ column vector of these variables as $\mathbf{z}$. Then, if $\mathbf{g}$ and $\mathbf{F}$ are evaluated at $\mathbf{z}=\mathbf{z}_{0}$, we can write the gradient near $\mathbf{z}_{0}$ as:

$$
\frac{\partial E^{\mathrm{UC}}}{\partial \mathbf{z}}=\mathbf{g}\left(\mathbf{z}_{0}\right)+\mathbf{F}\left(\mathbf{z}_{0}\right)\left(\mathbf{z}-\mathbf{z}_{0}\right) .
$$

Setting $\frac{\partial E^{\mathrm{UC}}}{\partial \mathbf{z}}=0$, we solve

$$
\mathbf{g}\left(\mathbf{z}_{0}\right)+\mathbf{F}\left(\mathbf{z}_{0}\right)\left(\mathbf{z}-\mathbf{z}_{0}\right)=0
$$

for $\mathbf{z}$. Since $\mathbf{F}$ is real and symmetric, it has only real eigenvalues, defined by

$$
\mathbf{F v}(n)=\lambda(n) \mathbf{v}(n), \quad n=1, \ldots, 3 N+9 .
$$

Then, the solution of (B2) is given by

$$
\mathbf{z}-\mathbf{z}_{0}=-\sum_{n=1}^{3 N+9} \frac{\mathbf{g}\left(\mathbf{z}_{0}\right)^{T} \mathbf{v}(n)}{\lambda(n)} \mathbf{v}(n) .
$$

Particularly if some of the eigenvalues are small, the coefficient $\left[\mathbf{g}\left(\mathbf{z}_{0}\right)^{T} \mathbf{v}(n)\right] / \lambda(n)$ may be large, and the resultant change in the variables, $\mathbf{z}-\mathbf{z}_{0}$, will be large. Large displacements are inconsistent with the validity of Eq. (B1) only for small displacements. In this case the magnitude, $\left\|\mathbf{z}-\mathbf{z}_{0}\right\|$ from Eq. (B3) is scaled to some given maximum. The fractional coordinates and lattice parameters are displaced accordingly to give a new configuration $\mathbf{z}_{0}^{\prime}$. Equation (B3) is then re-evaluated for the new reference configuration $\mathbf{z}_{0}^{\prime}$, and a new displacement is obtained. This iterative process is repeated until $\|\mathbf{g}\|$ falls below a given tolerance, or $\left\|\mathbf{z}-\mathbf{z}_{0}\right\|$ falls below a given tolerance or the predicted energy change, $\left|E^{U C}(\mathbf{z})-E^{U C}\left(\mathbf{z}_{0}\right)\right|$, falls below a given tolerance. The geometry is then taken to be optimized.

Note that all fractional coordinates and all lattice parameters are free variables in this optimization, so that the 
symmetry of the lattice is free to change during the optimization. However, the number of atoms in the unit cell is fixed.

${ }^{1}$ F. Cortés-Guzmán and R. F. W. Bader, Chem. Phys. Lett. 379, 183 (2003).

${ }^{2}$ D. W. Zhang and J. Z. H. Zhang, J. Chem. Phys. 119, 3599 (2003).

${ }^{3} \mathrm{~W}$. Li and S. Li, J. Chem. Phys. 122, 194109 (2005).

${ }^{4}$ D. G. Fedorov and K. Kitaura, J. Chem. Phys. 121, 2483 (2004).

${ }^{5}$ D. G. Fedorov and K. Kitaura, J. Chem. Phys. 122, 194109 (2005).

${ }^{6} \mathrm{~K}$. Babu and S. R. Gadre, J. Comput. Chem. 24, 484 (2003).

${ }^{7}$ W. Yang and T. Lee, J. Chem. Phys. 103, 5674 (1995).

${ }^{8}$ Y. Mei, C. Ji, and J. Z. H. Zhang, J. Chem. Phys. 125, 094906 (2006).

${ }^{9}$ X. H. Chen, D. W. Zhang, and J. Z. H. Zhang, J. Chem. Phys. 120, 839 (2004).

${ }^{10}$ X. H. Chen and J. Z. H. Zhang, J. Chem. Phys. 120, 11386 (2004).

${ }^{11}$ X. H. Chen and J. Z. H. Zhang, J. Theor. Comp. Chem. 3, 277 (2004).

${ }^{12}$ V. Deev and M. A. Collins, J. Chem. Phys. 122, 154102 (2005).

${ }^{13}$ M. A. Collins and V. A. Deev, J. Chem. Phys. 125, 104104 (2006).

${ }^{14}$ M. A. Addicoat and M. A. Collins, J. Chem. Phys. 131, 104103 (2009).

${ }^{15}$ R. P. A. Bettens and A. M. Lee, J. Phys. Chem. A 110, 8777 (2006).

${ }^{16}$ W. Li, S. Li, and Y. Jiang, J. Phys. Chem. A 111, 2193 (2007).

${ }^{17}$ J. M. Mullin, L. B. Roskop, M. A. Collins, and M. S. Gordon, J. Phys. Chem. A 113, 10040 (2009).

${ }^{18}$ M. S. Gordon, J. M. Mullin, S. R. Pruitt, L. B. Roskop, L. V. Slipchenko, and J. A. Boatz, J. Phys. Chem. B 113, 9646 (2009).

${ }^{19}$ H. M. Netzloff and M. A. Collins, J. Chem. Phys. 127, 134113 (2007).

${ }^{20}$ T. J. Frankcombe and M. A. Collins, Phys. Chem. Chem. Phys. 13, 8379 (2011).

${ }^{21}$ V. R. Saunders, R. Dovesi, and C. Roetti, CRYSTAL03 User's Manual (Torino, 2003)

${ }^{22}$ C. Pisani, M. Busso, G. Capecchi, S. Casassa, R. Dovesi, L. Maschio, C. Zicovich-Wilson, and M. Schütz, J. Chem. Phys. 122, 94113 (2005).

${ }^{23}$ P. Y. Ayala and G. E. Scuseria, J. Chem. Phys. 110, 3660 (1999).

${ }^{24}$ G. E. Scuseria, J. Chem. Phys. A 103, 4782 (1999).
${ }^{25}$ G. E. Scuseria and P. Y. Ayala, J. Chem. Phys. 111, 8330 (1999).

${ }^{26}$ P. Y. Ayala, K. N. Kudin, and G. E. Scuseria, J. Chem. Phys. 115, 9698 (2001).

${ }^{27}$ P. Giannozzi, S. d. Gironcoli, P. Pavone, and S. Baroni, Phys. Rev. B 43, 7231 (1991).

${ }^{28}$ See supplementary material at http://dx.doi.org/10.1063/1.3581845 for examples of the fragmentation process, optimized geometries, crystal energies, and differences in phonon frequencies.

${ }^{29}$ A. J. Stone, The Theory of Intermolecular Forces (Clarendon, Oxford, 1996).

${ }^{30}$ A. J. Stone, J. Chem. Theory Comput. 1, 1128 (2005).

${ }^{31}$ B. T. M. Willis and A. W. Pryor, Thermal Vibrations in Crystallography (Cambridge University Press, Cambridge, 1975).

${ }^{32}$ V. F. Sears, Neutron News 3, 26 (1992).

${ }^{33}$ NIST Center for Neutron Research, Neutron scattering lengths, see http://www.ncnr.nist.gov/resources/n-lengths/list.html

${ }^{34}$ G. Nilsson and G. Nelin, Phys. Rev. B 6, 3777 (1972).

${ }^{35} \mathrm{G}$. Dolling, Inelastic Scattering of Neutrons in Solids and Liquids (IAEA, Vienna, 1963), vol. II, p. 37.

${ }^{36}$ J. L. Warren, R. G. Wenzel, and J. L. Yarnell, Inelastic Scattering of Neutrons (IAEA, Vienna, 1965), Vol. I, p. 361.

${ }^{37}$ J. L. Warren, J. L. Yarnell, G. Dolling, and R. A. Cowley, Phys. Rev. 158, 805 (1967).

${ }^{38}$ D. Strauch and B. Dorner, J. Phys. Condens. Matter 5, 6149 (1993).

${ }^{39}$ O. Sode, M. Keçeli, S. Hirata, and K. Yagi, Int. J. Quant. Chem. 109, 1928 (2009).

${ }^{40}$ G. J. O. Beran and K. Nanda, J. Phys. Chem. Lett. 1, 3480 (2010).

${ }^{41}$ S. J. Nolan, P. J. Bygrave, N. L. Allan, M. J. Gillan, S. Binnie, and F. R. Manby, in Accurate Condensed-Phase Quantum Chemistry, edited by F. R. Manby (CRC Press, Boca Raton, 2011), p. 85.

${ }^{42}$ H. Stoll, Chem. Phys. Lett. 191, 548 (1992).

${ }^{43}$ H. Stoll, Phys. Rev. B 46, 6700 (1992).

${ }^{44}$ B. Paulus, Phys. Rep. 428, 1 (2006).

${ }^{45}$ K. C. Thompson, M. J. T. Jordan, and M. A. Collins, J. Chem. Phys. 108, 564 (1998). 